\title{
ACOUSTIC PHONON ACTIVATED AND ASSISTED TUNNELLING IN Ge:Sb,P
}

\author{
N. Žurauskiené and A. Dargys
}

Semiconductor Physics Institute, A. Goštauto 11, 2600 Vilnius, Lithuania

The influence of lattice vibrations on the field ionization rate of shallow donors in germanium at low lattice temperatures is investigated experimentally and theoretically. The role played by acoustic phonons in the tunnelling of electrons from the ground donor level (phonon assisted tunnelling) and through the excited donor levels (phonon activated tunnelling) is considered. Both processes are shown to enhance the tunnelling rate.

PACS numbers: 71.70.Ms, 73.40.Gk, 79.70.+q

\section{Introduction}

In solids the lattice vibrations can participate in electron and hole tunnelling through the potential barriers formed by conduction or valence band edges. Up till now most of the investigations were devoted to impurity field ionization where optical rather than acoustical phonons are of importance [1]. The energy of the optical phonons weakly depends on their wave vector. This explains why experimental observation as well as theoretical interpretation is simpler in the optical phonon case. The participation of acoustical phonons in the tunnelling process is expected to become important at low lattice temperatures and low barriers, of the order of, or smaller than, the optical phonon energy. Recently we have investigated the influence of acoustic phonons on the field ionization of phosphorus atoms in a Si lattice [2] and of carbon atoms in a GaAs lattice [3].

Below the role of acoustic phonons played in the field ionization of phosphorus and antimony atoms in ultrapure germanium single crystals is investigated. Two processes associated with the acoustic phonons (phonon assisted tunnelling and phonon activated tunnelling) are considered to explain the experimental results.

\section{Results}

According to the specification and our earlier measurements [4] the investigated single crystal of Ge contained mainly two shallow donor species (phosphorus and antimony) of the total concentration $N^{\mathrm{P}}+N^{\mathrm{Sb}} \approx 10^{13} \mathrm{~cm}^{-3}$. Measurements were performed with the transient tunnelling spectrometer [5] which, for this purpose, was tuned to a special measuring regime $[2,3]$. Periodic and linearly ramped 
voltage pulses were applied to the investigated samples in a form of mesas. When the voltage amplitude reached a certain value, a fast tunnelling of electrons out of the donors was observed as an exponential growth of the current in the load resistor. To find the variation of the tunnelling rate with temperature, the lattice temperature was slowly increased and the tunnelling current was sampled at a predetermined voltage amplitude in the ramp. The current sampled in this way was proportional to the donor field ionization rate $W$ at temperature $T$ and at the electric field $E$. The latter in our experiments ranged from 1450 to $1700 \mathrm{~V} / \mathrm{cm}$. To avoid multivalley effects, the electric field was directed parallel to $\langle 100\rangle$ crys-

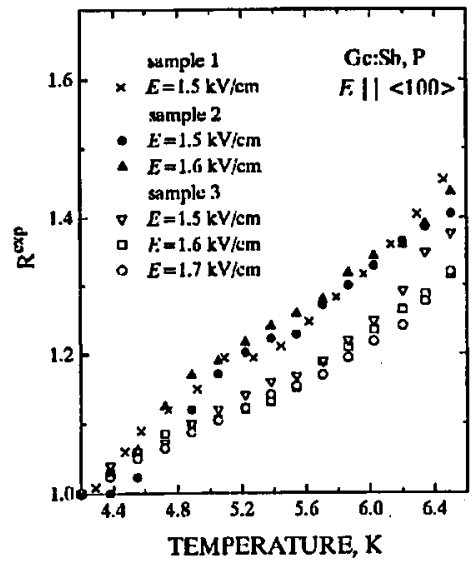

Fig. 1. Experimental values of the tunnelling rate normalized to the value at $4.2 \mathrm{~K}$ as a function of lattice temperature for three $n$-Ge:Sb,P samples.

tallographic direction. The points in Fig. 1 show the normalized tunnelling rate $R^{\exp }=W(T) / W(4.2)$ as a function of lattice temperature for three samples. The highest experimental temperature was limited by an influence on the total current of free, thermal electrons in the conduction band. From Fig. 1 it follows that the effect of temperature on the tunnelling rate is not large.

\section{Discussion}

Two mechanisms will be considered in explaining the observed increase in the tunnelling rate. The first, phonon assisted tunnelling mechanism $[6,7]$, assumes that an electron tunnels from the ground level through thermally vibrating potential barrier of an impurity atom. The second, phonon activated tunnelling mechanism [8], assumes that the electron at first is thermally excited to a higher-lying impurity level and only then tunnels through a more transparent donor barrier. To find the contribution of the phonon assisted tunnelling the acoustic deformation-potential interaction of the donor electron with the lattice vibrations was considered as in [2,3]. Figure 2 shows the ratio $R^{\text {assis }}=W(T) / W(0)$ calculated with the parameter values which are typical of Ge:Sb. From the figure it follows that even at the highest measurement temperature, $T=6.5 \mathrm{~K}$, the con- 


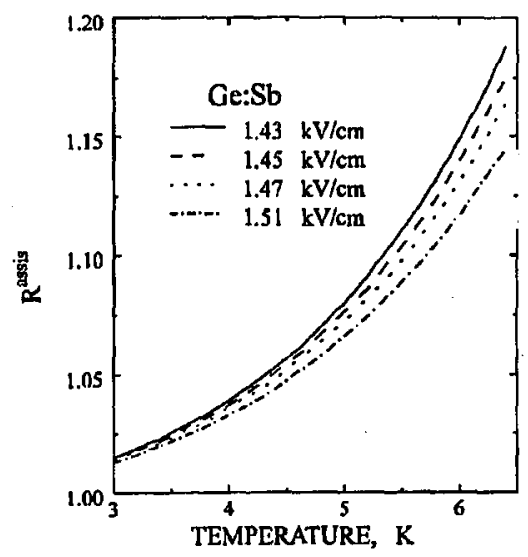

Fig. 2. Normalized to zero temperature tunnelling rate versus lattice temperature for the phonon assisted mechanism.

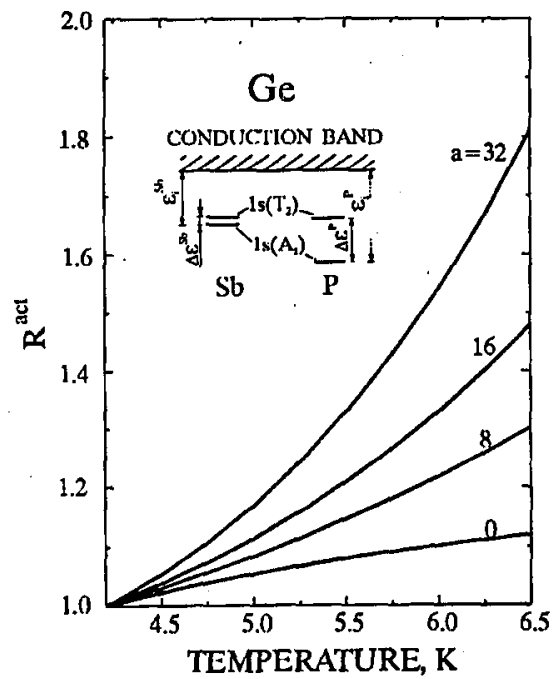

Fig. 3. Normalized to $T=4.2 \mathrm{~K}$ tunnelling rate versus lattice temperature for the phonon activated tunnelling mechanism calculated at various parameter $a$ values. The inset shows the lowest energy level structure of $\mathrm{Sb}$ and $\mathrm{P}$ atoms which was used in the calculation. Ionization energy: $\varepsilon_{i}^{\text {Sb }}=10.45 \mathrm{meV}, \varepsilon_{i}^{\mathbf{P}}=12.88 \mathrm{meV}$. Energy difference: $\Delta \varepsilon^{\mathrm{Sb}}=0.46 \mathrm{meV}, \Delta \varepsilon^{\mathrm{P}}=2.82 \mathrm{meV}$.

tribution of the phonon assisted tunnelling is rather small, $W(6.5) / W(4.2) \approx 1.1$, and cannot explain the experiment, where larger variations were observed.

Now, we shall stop on the activated tunnelling. We shall assume that electron thermal transitions between the ground $1 s\left(A_{1}\right)$ and the first excited $1 s\left(T_{2}\right)$ donor levels (see the inset in Fig. 3), due to absorption and emission of acoustic phonons, 
are fast in comparison with a characteristic electron tunnelling time (in our case about $10 \mathrm{~ns}$ ), and that the donor potential barriers are stationary (no phonon assistance). Then the ratio of the tunnelling rates at temperatures $T$ and $4.2 \mathrm{~K}$ is [9]

$$
\begin{aligned}
& R^{\mathrm{Sb}+\mathrm{P}}=\frac{W_{\mathrm{eff}}^{\mathrm{Sb}}(T)+a W_{\mathrm{eff}}^{\mathrm{P}}(T)}{W_{\mathrm{eff}}^{\mathrm{Sb}}(4.2)+a W_{\mathrm{eff}}^{\mathrm{P}}(4.2)}, \\
& W_{\mathrm{eff}}^{j}(T)=\frac{\exp \left(-\alpha_{T 2}^{j} / E\right)\left(g_{2} / g_{1}\right) \exp \left(-\Delta \varepsilon^{j} / k T\right)+\exp \left(-\alpha_{A 1}^{j} / E\right)}{\left(g_{2} / g_{1}\right) \exp \left(-\Delta \varepsilon^{j} / k T\right)+1},
\end{aligned}
$$

where $W_{\text {eff }}^{j}(T)$ is the effective field ionization rate for donor species $j=\mathrm{Sb}$ or P. Levels $1 s\left(A_{1}\right)$ and $1 s\left(T_{2}\right)$ have degeneracies $g_{1}=1$ and $g_{2}=3$, respectively, and the energetic distance $\Delta \varepsilon^{j}=\varepsilon^{j}\left(A_{1}\right)-\varepsilon^{j}\left(T_{2}\right)$. The coefficient $a$ in Eq. (1) is equal to $a=\left(N^{\mathrm{P}} / N^{\mathrm{Sb}}\right) /\left(C^{\mathrm{P}} / C^{\mathrm{Sb}}\right)$, where $C^{\mathrm{P}} / C^{\mathrm{Sb}}$ is the ratio of preexponential tunnelling factors in the respective tunnelling rates: $W^{j}=\left(C^{j} / E\right) \exp \left(-\alpha^{j} / E\right)$. The lines in Fig. 3 show calculated with Eqs. (1) and (2) the normalized rates when $a=0,8,16$ and 32 . The required parameter values $\left(\alpha_{A 1}^{\mathrm{Sb}}=25.1 \mathrm{kV} / \mathrm{cm}\right.$, $\alpha_{T 2}^{\mathrm{Sb}}=23.46 \mathrm{kV} / \mathrm{cm}, \alpha_{A 1}^{\mathrm{P}}=34.34 \mathrm{kV} / \mathrm{cm}$ and $\left.\alpha_{T 2}^{\mathrm{P}}=23.71 \mathrm{kV} / \mathrm{cm}\right)$ were found from the hydrogenic formula using appropriate values of the ionization energies for $1 s\left(A_{1}\right)$ and $1 s\left(T_{2}\right)$ levels. The line $a=0$ corresponds to the case when $\mathrm{Sb}$ atoms are important only. From Fig. 3 it follows that $a$ is close to 16 for samples 1 and 2 and close to 8 for sample 3 , and that the phosphorus atoms give rather large contribution in the variation of $R^{\text {act }}$ versus $T$.

In summary, in the temperature range from $4.2 \mathrm{~K}$ to $6.5 \mathrm{~K}$ the growth of about $40 \%$ in the tunnelling rate was observed in Ge:Sb,P. The growth could be explained by the sum of two processes: the phonon assisted tunnelling and the phonon activated tunnelling.

\section{References}

[1] A.N. Georgobiani, P. Pipinys, Tunnel Phenomena in Semiconductor Luminescence, Mir, Moskva 1994 (in Russian).

[2] A. Dargys, N. Žurauskiené, J. Appl. Phys. 78, 4802 (1995).

[3] A. Dargys, Š. Kudžmauskas, S. Žurauskas, J. Phys., Condens. Matler 7, 8967 (1995).

[4] A. Dargys, S. Žurauskas, N. Žurauskiene, Phys. Lett. A 157, 419 (1991).

[5] A. Dargys, S. Žurauskas, N. Žurauskienè, Appl. Phys. A 52, 13 (1991).

[6] V.N. Abakumov, V. Karpus, V.I. Perel, I.N. Yassievich, Fiz. Tverd. Tela 30, 2498 (1988).

[7] Š. Kudžmauskas, Liet. Fiz. Žurn. 34, 519 (1994) [Lithuanian J. Phys. 34, 445 (1994)].

[8] A. Dargys, N. Žurauskienè, S. Žurauskas, Phys. Status Solidi B 162, 183 (1990).

[9] N. Žurauskiené, Ph.D. Thesis, Semicond. Phys. Inst., Vilnius 1995. 